\title{
Best for Braille Users: A Comparison of Mapping Software
}

\author{
Ashna Abdulrahman Kareem Zada \\ Faculty of Informatics, Department of Cartography and Geoinformatics, Eötvös Loránd University, Hungary, Ashna Abdulrahman \\ Kareem Zada - ashnakareem88@gmail.com
}

Keywords: Software, Maps, GIS, Sightless

\begin{abstract}
:
Mapping software refers to a type of computer software that allows the user to graphically represent, analyze, and model large quantities of data in either a static or dynamic format, allowing the user to convey a geographical information. It is also a visual tool that allows specialists to communicate and share spatial information easily. The researcher found that the three most significant aspects of successful map creation were those that were visual, spatial, and those that allowed for effective communication. Within the context of this study, mapping software is explored within the context of the benefits of different mapping software choices as they pertain to the benefits that those who are blind or visually impaired may receive from the software itself. The main purpose of the study was to explore the extent to which persons with low vision, visual impairment, or blindness found mapping software to be useful. Moreover, the researcher investigated the existing advantages and disadvantages of using different mapping software options and compared the features of those programs with the features of the programs that have been considered to be the best for use with Braille. The results show that ArcGIS offers a powerful way to visualize data and gain understanding and insights that may have been hidden or less accessible in other software choices.
\end{abstract}

